\title{
Entwicklung eines Fragebogens zur Selbst- und Fremdeinschätzung von OPD-KJ-Konflikten durch Patient innen und ihre Therapeut_innen
}

Inge Seiffge-Krenke \& Fabian Escher

Psychologisches Institut der Universität Mainz

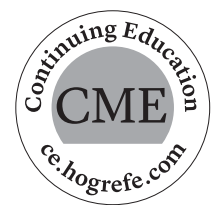

\begin{abstract}
Zusammenfassung: Der OPD-KJ-Konfliktfragebogen legt eine Version zur Erfassung der Bewältigungsmodi unbewusster Konflikte gemäß der Operationalisierten Psychodynamischen Diagnostik (OPD-KJ) für Patient_innen und ihre Therapeut_innen vor. Zur Auswahl der Items für den Konfliktfragebogen wurden Expertenratings und psychometrische Gütekriterien, basierend auf einer Stichprobe von 427 Jugendlichen (53.6\% Patienten) und 44 Therapeut_innen, kombiniert. Die Kurzversion enthält 28 Items, die sieben intrapsychische Konflikte und ihre aktiven bzw. passiven Bewältigungsmodi umfasst (Gesamt ICC $=.855 ; p<.001$ ). Es ergaben sich erwartbare Unterschiede zwischen gesunden und klinisch auffälligen Jugendlichen mit einem höheren Konfliktniveau der ambulanten Patient_innen. Ein Vergleich der Therapeutenversion mit zwei verschiedenen Methoden (Befunderhebung und Konfliktfragebogen) erbrachte übereinstimmende Einschätzungen an der gleichen Patientin sowohl in der Experteneinschätzung (Gesamt ICC $=.861 ; p<.001$ ) als auch aus der Sicht von 52 Teilnehmer_innen von zwei OPD-KJ-Trainingsgruppen (Gesamt ICC $=.825 ; p<.001)$.
\end{abstract}

Schlüsselwörter: OPD-KJ, Konfliktachse, Fragebogen, Jugendliche, Therapeut_innen

Psychodynamic conflicts: Development of a questionnaire for the assessment by patients and their therapists

Abstract: The OPD-CA conflict questionnaire represents a means of recording modes of coping with unconscious conflicts according to the Operationalized Psychodynamic Diagnostics (OPD-CA) for patients and their therapists. To select the items for the conflict questionnaire, we combined the expert ratings and psychometric quality criteria using a sample of 427 adolescents ( $53.6 \%$ patients) and 44 therapists. The short version contains 28 items, which include 7 intrapsychic conflicts and their active and passive coping modes (total ICC $=.855 ; p<.001$ ). We found expected differences between healthy and clinically abnormal adolescents with a higher level of conflicts among outpatients. A comparison of the therapist's version with two different methods (assessment according to the sheet in OPD-CA and the conflict questionnaire) produced consistent assessments of the same patient both in the expert assessment (total ICC $=.861 ; p<.001$ ) and from the point of view of 52 participants in two OPD-KJ training courses (total ICC $=.825 ; p<.001$ ).

Keywords: OPD-CA, conflict questionnaire, adolescent patients, therapists

\section{Einleitung}

Die gegenwärtig vorliegenden nosologischen Klassifikationen, wie etwa die ICD-10 (International Statistical Classification of Diseases and Related Health Problems), orientieren sich an Symptomen, indem sie eine Vielzahl klinischer Störungsbilder nach symptomatischen Merkmalen gruppieren. Für die diagnostische und klinische Arbeit in der Kinder- und Jugendpsychiatrie und Psychotherapie ist es darüber hinaus wichtig, zusätzliche Informationen zu haben, wie etwa belastende Konflikte, die das Leben von Kindern und Jugendlichen beeinträchtigen und die Entwicklung hemmen, die strukturellen Vor- aussetzungen sowie die Therapiemotivation, die wichtige Informationen für die Indikation zu einer stationären oder ambulanten Therapie und das weitere therapeutische Vorgehen enthalten.

Unbewusste Konflikte nehmen eine zentrale Stellung innerhalb der psychodynamischen Störungstheorie ein (Freud, 1916/1917). Dem Freud'schen Krankheitsmodell lag die Triebtheorie zugrunde, d.h., Konflikte zwischen den Triebwünschen und äußeren Hemmnissen waren zentral. Diese Zentrierung auf das Triebgeschehen wird aus heutiger Sicht nicht mehr für adäquat gehalten (Benecke \& Brauner, 2017). Stattdessen wird in psychodyna- 
mischen Konflikttheorien eher auf basale Motivsysteme Bezug genommen wie etwa Bindung (Bowlby, 1969), Selbstwert (Kohut, 1979) und Identität (Erikson, 1966). Der Kern des Modells blieb allerdings auch in modernen Konfliktmodellen erhalten: Ein Scheitern der Integration der basalen Bedürfnisse und Motive führt zu dynamisch unbewussten Konflikten. Sie machen das Individuum vulnerabel für die spätere Entstehung psychischer Störungen (Seiffge-Krenke, 2020a).

Orientiert an psychodynamischen Konzepten wird in der Operationalisierten Psychodynamischen Diagnostik im Kindes- und Jugendalter (Arbeitsgruppe OPD-KJ-2, 2020) als Ursache von Störungen ein intrapsychischer, zeitlich überdauernder Konflikt angenommen, bei dem unvereinbare Bestrebungen aufeinanderprallen, die in einem aktiven bzw. passiven Modus "gelöst" werden. Entscheidendes Kriterium ist, dass sich der intrapsychische Konflikt in verschiedenen Alltagsbereichen beobachten lässt und die Weiterentwicklung des Kindes oder Jugendlichen hemmt oder blockiert. Die Integration dieser widersprüchlichen Bestrebungen gelingt nicht und wirkt sich in einer spezifischen Färbung der Beziehungsgestaltung in unterschiedlichen Alltagsbereichen (z.B. Schule, Elternhaus, Peers) aus, wobei zwischen einem aktiven oder passiven Modus der Verarbeitung des jeweiligen Konflikts unterschieden wird. Ein Konflikt im Sinne der OPD-KJ ist also durch die sechs Merkmale (1) intrapsychisch, (2) zeitlich überdauernd, (3) entwicklungsbehindernd (4) aktiver bzw. passiver Verarbeitungsmodus, (5) beobachtbar in verschiedenen Alltagsbereichen und (6) Auswirkungen auf die Beziehungsgestaltung gekennzeichnet.

Sieben Konfliktthemen sind $\mathrm{zu}$ unterscheiden, die neben dem ödipalen Thema, das noch für Freud für die Symptomentstehung so wichtig war, der konzeptuellen Weiterentwicklung entsprechend bindungsbezogene, objektbeziehungstheoretische und selbstpsychologische Konfliktthemen aufnehmen: Der Nähe-Distanz-Konflikt erfasst im aktiven Modus Angst vor Nähe, übersteigerte emotionale Unabhängigkeit, im passiven Modus dagegen Angst vor Trennung und ständige Suche nach engen Beziehungen. Im Konflikt Unterwerfung vs. Kontrolle findet sich im aktiven Modus ein ständiges Aufbegehren gegen Pflichten, im passiven Modus Gefügigkeit und Unterordnung. Beim Konflikt Selbst versorgen vs. Versorgtwerden finden wir im aktiven Modus Selbstversorgung und Aufopferung für andere, im passiven Modus ein anklammerndes, parasitäres Verhalten. Beim Selbstwertkonflikt steht die Regulierung des Selbstwertes in Beziehungen im Vordergrund, zum einen im aktiven Modus durch grandiose Selbstüberschätzung, zum anderen im passiven Modus mit sehr geringem Selbstwert. Der Schuldkonflikt enthält im aktiven Modus Entwertungen der familiä- ren Beziehungen, im passiven Modus eine überzogene Treuebindung an die Eltern. Beim ödipalen Konflikt wird im aktiven Modus Sexualität überbetont, während Kinder und Jugendliche im passiven Modus sachlich und eher sexuell unattraktiv und uneindeutig erscheinen. Beim Identitätskonflikt finden wir im aktiven Modus eine unkritische Übernahme wechselnder Identifizierungen, im passiven Modus große Orientierungs- und Ratlosigkeit.

Inzwischen liegen zahlreiche Studien zur Reliabilität und Validität der Konfliktachse, erhoben mit dem Befundbogen (Arbeitsgruppe OPD-KJ-2, 2020, S. 390), vor (Rathgeber, Sommer \& Seiffge-Krenke, 2014; SeiffgeKrenke, Mayer \& Winter, 2011; Stefini et al., 2013; Winter, Jelen, Pressel, Lenz \& Lehmkuhl, 2011). Grundlage für die Einschätzung mit dem Befundbogen ist das OPD-KJ-Interview (Arbeitsgruppe OPD-KJ-2, 2020, S. 367-382). Das Manualisierungskapitel (Arbeitsgruppe OPD-KJ-2, 2020 S. 139-196) erleichtert die Einstufung, indem es für jeden Konflikt, und jeweils gegliedert anhand der Altersfenster 1-3 und betroffenem Lebensbereich, den aktiven und passiven Modus der Verarbeitung der sieben Konflikte erläutert. Beim OPD-KJ-Konfliktrating wird anhand des Manuals und mithilfe des Befundbogens (Arbeitsgruppe OPD-KJ-2, 2020, S. 390) der Konflikt (und dessen Bewältigungsmodus) auf der Basis der Aussagen der Patientin bzw. des Patienten im OPD-KJ-Interview, aus der Beobachtung der Szene sowie aus biografischen Angaben der Eltern und möglicherweise einer Spielbeobachtung eingestuft. Für die Therapeutin oder den Therapeuten bzw. die Diagnostikerin oder den Diagnostiker liegen daher umfangreiche Informationen vor, aus deren Integration man ein schon länger vorliegendes entwicklungsbehinderndes Thema, einen Konflikt, ableiten kann. In der Regel geschieht die Erhebung dieser Informationen in mehreren diagnostischen Sitzungen, wobei man anfangs eine Konflikthypothese bildet, die man im Verlauf weiterer Sitzungen in Bezug auf die vorliegenden Indikatoren überprüft. Grundsätzlich wird das Vorhandensein bzw. Nichtvorhandensein eines Konfliktes und seine entsprechende Ausprägung bei jeder Patientin bzw. jedem Patienten für jeden Konflikt geratet.

Die reliable Einstufung setzt entsprechende Trainings voraus. In der Regel werden drei Trainingskurse angeboten, ein Grundkurs und zwei Aufbaukurse, in denen die Einstufung aller Achsen mit einem Gesamtumfang von 35 Stunden eingeübt und zertifiziert wird. Die Trainings zum Erlernen der Einstufung erwiesen sich als wirksam (Seiffge-Krenke et al., 2011). In dieser Studie wurde in einer Trainingsgruppe $(N=20)$ die Beurteilerübereinstimmung (ICC) über 18 videografierte Patient_innen auf der Grundlage des Befundbogens zu mehreren Messzeitpunkten erhoben. Die Interrater-Reliabilität betrug ICC $=.76$ 
nach dem ersten Training und verbesserte sich über die Zeit hinweg signifikant um .10.

Eine weitere empirische Studie an 80 Forschungszwillingen belegte, dass bei klinisch unauffälligen Kindern und Jugendlichen die gleichen Konfliktthemen vorkommen, allerdings in einer deutlich geringeren Ausprägung. Die Mittelwerte fielen in einzelnen Konflikten und im Gesamtkonfliktwert in der altersgleichen klinischen Gruppe zwei- bis dreimal höher aus, was für die starke Beschäftigung und Beeinträchtigung durch die Konflikte spricht (Seiffge-Krenke et al., 2014). Nach OPD-KJ (Arbeitsgruppe OPD-KJ-2, 2020) können im Prinzip alle Konfliktthemen bei allen Diagnosen vorkommen. Dies ließ sich weitgehend bestätigen. Man fand bei Patient_innen mit externalisierenden Störungen lediglich etwas häufiger den Konflikt Unterwerfung vs. Kontrolle, bei Patient_innen mit internalen Störungen hingegen häufiger den Versorgungskonflikt (Winter et al., 2011). Die Erhebung von Daten zur Konflikt- und Strukturachse erwiesen sich als wichtige Hilfe für die Indikation und Therapieplanung bei stationären und ambulanten Patient_innen (Seiffge-Krenke, Mayer, Rathgeber \& Sommer, 2013), insbesondere im Kontext von Strukturdefiziten (SeiffgeKrenke, Fliedl \& Katzenschläger, 2013).

\section{Fragestellung}

Aufgrund des recht hohen Aufwandes entstand die Idee, ein ökonomisches Verfahren in Fragebogenform zu entwickeln. Für die Erwachsenen-OPD liegen bereits verschiedene Selbstbeurteilungsverfahren vor: Für die Strukturachse gibt es einen Fragebogen in der Kurzversion (OPD-SFK; Ehrenthal et al., 2015), für die OPD-Beziehungsachse gibt es ebenfalls ein Selbstbeurteilungsinstrument (OPD-BQS; Zimmermann, Stasch, Grande, Schauenburg \& Cierpka, 2014). Benecke et al. (2018) haben des Weiteren die Entwicklung und Validierung eines OPD-Konfliktfragebogens (OPD-KF) für Erwachsene und Hold, Benecke und Schüßler (2015) ein Selbstberichtsverfahren für Jugendliche beschrieben. Für den Bereich der OPD-KJ wurde inzwischen ein Strukturfragebogen entwickelt (Schrobildgen, Goth, Weissensteiner, Lazari \& Schmeck, 2019).

Die Entwicklung des OPD-KJ-Konfliktfragebogens folgt der Logik der Konfliktratings wie sie im Befundbogen operationalisiert wurden: Unbewusste Konflikte per se können nicht direkt/bewusst erfasst werden, also auch nicht per Fragebogen. Beim regulären OPD-KJ-Konfliktrating anhand des Manuals mithilfe des Befundbogens (OPDKJ-2, 2020, S. 390) liegen zur Einstufung der Konflikte (und ihrer Bewältigungsmodi) wie beschrieben umfang- reiche Informationen vor, die in mehreren diagnostischen Sitzungen erhoben werden. Das Vorgehen bei der Beantwortung des Konfliktfragebogens folgt derselben Logik, denn die Diagnostikerin bzw. der Diagnostiker oder die Therapeutin bzw. der Therapeut verfügen auch hier über umfangreiche Vorabinformationen und sollten den Konfliktfragebogen erst am Ende der diagnostischen Phase einstufen. Die Items des Fragebogens repräsentieren daher Aussagen, die als typisch für jemanden angesehen werden, bei dem der jeweilige Konflikt mehr oder weniger stark ausgeprägt ist (jeweils getrennt für passiven und aktiven Modus).

Zunächst war es notwendig, die Einschätzung der Therapeutin bzw. des Therapeuten oder der Diagnostikerin bzw. des Diagnostikers zu erhalten, denn sie haben zahlreiche Informationen, anhand derer sie einen möglichen intrapsychischen entwicklungsbehindernden Konflikt einstufen können, der die Indikation zur psychodynamischen Therapie und zur präzisen Behandlungsplanung erleichtern kann. Zugleich war es uns wichtig, die Einschätzung der Patient_innen zu erhalten, also ein Selbstbeurteilungsinstrument $\mathrm{zu}$ erstellen. Es erschien uns sinnvoll, nur bei der ältesten Altersstufe (Altersstufe 3 = 13-18 Jahre), also Jugendlichen, ein solches Selbstbeurteilungsinstrument einzusetzen. Das Reflexionsniveau über sich und andere ist abhängig vom Strukturniveau, aber auch vom Alter. Die entwicklungspsychologische Forschung zeigt, dass Jugendliche im Vergleich zu jüngeren Altersstufen zu einem höheren Reflexionsniveau in der Lage sind und psychologische Charakteristiken bei der Selbst- und Fremdbeschreibung verwenden (Seiffge-Krenke, 2020b).

Um die Erfassung des intrapsychischen Konflikts ökonomischer zu gestalten und eine standardisierte Erhebung zu ermöglichen, haben wir einen multiperspektivischen Fragebogen für die Altersstufe 3 nach OPD-KJ-2 erstellt, der prinzipiell, in der Therapeutenversion, auch für jüngere Altersstufen einschätzbar ist. Wir konzentrieren uns in diesem Beitrag auf die Entwicklung des Konfliktfragebogens in der Selbst- und Fremdeinschätzung für jugendliche Patient_innen der Altersstufe 3 und ihre Therapeut_innen. Die Entwicklung des Konfliktfragebogens erfolgt in vier aufeinander bezogene Stufen, die im Folgenden genauer beschrieben werden:

a. Konstruktion und Reduktion des Fragebogens

b. Kriteriumsvalidität

c. Konstruktvalidität

d. Diskriminante Validität

Dabei sollen unterschiedlichen Stichproben und Vorgehensweisen jeweils unterschiedliche Beiträge zur Aufklärung der Anwendbarkeit leisten, sie betreffen entsprechend unterschiedliche Aspekte der Validität. 


\section{Methode}

\section{Stichproben und Vorgehensweise bei Fragebogen-Konstruktion und Validierung}

In verschiedenen Phasen der Entwicklung des Konfliktfragebogens wurden unterschiedliche Stichproben von Jugendlichen und Kinder- und Jugendlichenpsychiater_innen bzw. Psychotherapeut_innen einbezogen, um die verschiedenen Fragestellungen der Validierung des Konfliktfragebogens zu beantworten.

\section{a) Die Konstruktion und Überprüfung der Langversion, Entwicklung einer Kurzversion}

Die Erstellung und Überprüfung der Langversion mit 42 Items erfolgte anhand der Daten von 121 jugendlichen Patient_innen und ihren 46 Therapeut_innen. Die jugendlichen Patient_innen $(M=16.29$ Jahre, $S D=1.95 ; 73.6 \%$ Mädchen) und ihre Therapeut_innen $(M=35.61$ Jahre, $S D=9.81 ; 91.2 \%$ weiblich) füllten die Selbst- und Fremdeinschätzung mittels des Konfliktfragebogens während der diagnostischen Phase und damit vor Beginn einer psychotherapeutischen Behandlung aus. Es wurden alle Patient_innen einbezogen (92\%), für die nach der Diagnostik anhand der ICD-10, die durch unabhängige Diagnostiker_innen durchgeführt worden war, eine Indikation für eine psychodynamische Behandlung gestellt worden war. Patient_innen mit neurologischen Beeinträchtigungen und schweren Entwicklungsbehinderungen wurden ausgeschlossen. Die häufigsten Diagnosekategorien der Patient_innen waren Angsterkrankungen und Belastungsstörungen (F40, 38.6\%), Affektive Störungen (F30, 22.6\%) und Essstörungen (F50, $21.3 \%$ ). Seltener waren Entwicklungsstörungen (F90, $14.4 \%)$ und Persönlichkeitsstörungen (F60, 3.1\%). Bei den Therapeut_innen handelt es sich um Therapeut_innen in psychodynamischer Weiterbildung mit einer durchschnittlichen Behandlungserfahrung von 2.32 Jahren $(S D=1.76)$. Die Therapeut_innen waren überwiegend weiblich $(91.2 \%)$ und hatten ein Durchschnittsalter von $35.61(S D=9.81)$.

In die Überprüfung der Langversion zur Erstellung einer Kurzversion wurden auch neun erfahrene OPD-KJ-Experten_innen aus dem Arbeitskreis ${ }^{1}$ einbezogen. Die Therapeutenlangversion wurde von diesen neun Expert_innen bzgl. ihrer inhaltlichen Stimmigkeit mit Operationalisierungen im Manual eingeschätzt.

\section{b) Kriteriumsvalidität: Überprüfung der Kurzversion und Standardisierung (Erstellung von $T$-Werten für Jugendliche und Therapeut_innen)}

Die dann erstellte Kurzversion mit 28 Items wurde anschließend von insgesamt $N=427$ (198 gesunde Jugendliche und 229 Patient_innen) beantwortet. Die 229 ambulanten Patient_innen hatten in einem Ausbildungsinstitut um eine ambulante Psychotherapie nachgesucht. Es handelt sich um drei Viertel weibliche ( $f \%=76.0, f=174)$ und knapp einem Viertel männliche Jugendliche $(f \%=24.0, f=55) \mathrm{mit}$ einem Durchschnittsalter von 16.96 Jahren $(S D=1.92)$. Diese Patient_innen befanden sich in der diagnostischen Phase; es war im Rahmen der ambulanten Sprechstunde eine eindeutige krankheitswertige Störung festgestellt worden, die eine Indikation für eine psychodynamische Psychotherapie darstellte. Die häufigsten Diagnosekategorien der Patient_innen waren Angsterkrankungen und Belastungsstörungen (F40, 35.2\%), Entwicklungsstörungen (F90, 28.7\%) und Affektive Störungen (F30, 22.5\%). Seltener waren Essstörungen (F50, 9.5\%) und Persönlichkeitsstörungen (F60, $4.1 \%)$.

Auf der Basis der Patientendaten wurden in einer Onlineerhebung Forschungszwillinge erhoben. Es hatten sich 300 klinisch unauffällige Jugendliche gemeldet, davon wurden 102 Jugendliche aussortiert, weil wir im Alter und Geschlecht möglichst große Ähnlichkeiten mit der Patientenstichprobe erzielen wollten. Daher wurden teilnehmende Jugendliche, die nicht als Forschungszwillinge infrage kamen sowie solche mit zu vielen fehlenden Werten in ihren Fragebögen aussortiert.

Dieses Vorgehen schien uns sinnvoll, weil insbesondere im ambulanten Psychotherapiebereich in der Regel mehr Patientinnen kommen, wie es auch bei unserer Patientenstichprobe der Fall war. Insgesamt nahmen 198 gesunde Jugendliche (Alter $=16.89 ; S D=1.95)$ an unserer Erhebung teil. Die gesunden Jugendlichen sind ebenfalls mehr Mädchen (68.2\%) als Jungen (31.3\%). Die Patient_innen kommen häufiger aus Einelternfamilien (44.2\%) als die gesunden Jugendlichen (26.3\%). Der Familienstand unterscheidet sich signifikant $\left(\chi^{2}=62.34 ; p<.001\right)$. Es bestehen keine signifikanten Unterschiede in Alter $(F[1,427]=4.35, p=.471$, $\left.\eta^{2}=.003\right)$ und Geschlecht $\left(\chi^{2}=5.74 ; p=.857\right)$.

Die 229 Patient_innen aus Studie b) und die 121 Patient_innen aus Studie a) stellen die Datengrundlage für die Erstellung der $T$-Werte für Jugendliche dar $(N=340)$. Die Grundlage für die Therapeuten-T-Werte stellen die 46 Therapeut_innen aus Studie a) dar. 


\section{c) Konstruktvalidität: Methodenvergleich Befundbogen vs. Konfliktfragebogen}

Die Kurzversion wurde von drei Expert_innen am Beispiel eines Videos einer Patientin eingeschätzt. Bislang liegen kaum Videos von Patient_innen aus ambulanten Behandlungssettings vor. Daher wurde als einheitliche Informationsgrundlage für die Einschätzung aller Therapeut_innen ein Video einer stationär aufgenommenen Jugendlichen ausgewählt, die an einer kombinierten Störung des Sozialverhaltens und der Emotionen (F92) litt und an einem 1-stündigen Interview nach dem OPD-KJ-Interviewleitfaden teilnahm. Sie wurde von einem männlichen Therapeuten interviewt. Drei erfahrene OPD-KJ-Trainer_innen ${ }^{2}$ schätzten dann unabhängig voneinander das Video anhand des Konfliktfragebogens ein. Auf der Basis des gleichen Patientinnen-Videos wurde dann ein Methodenvergleich vorgenommen. An diesem Vergleich der Konfliktachse, wie sie im Befundbogen im Manual der OPD-KJ-2 eingeschätzt werden kann, mit der 28-Items-Kurzform des Konfliktfragebogens nahmen 52 Teilnehmer_innen im Rahmen von OPD-KJ-Trainings teil. Die Kinder- und Jugendpsychiater innen bzw. -therapeut_innen (48 weiblich, 4 männlich) waren im Alter von 35 Jahren $(M=34.8 ; S D=6.65)$. Bei den Teilnehmer_innen handelte es sich um 34 Psycholog_innen und 18 Ärztinnen bzw. Ärzte (in Ausbildung befindlich bzw. mit abgeschlossener psychodynamischer Zusatzausbildung und wenige Teilnehmer_innen mit systemischem Hintergrund). Die Trainingsgruppen nahmen in Mainz, Deutschland $(N=25)$ und Salzburg, Österreich $(N=27)$ am dritten Teil der OPD-KJ-Trainings teil und bekamen den Bogen zur Befunderhebung und die Konfliktskalen in zufälliger Reihenfolge vorgelegt.

\section{d) Überprüfung der diskriminanten Validität}

Zur Überprüfung der diskriminanten Validität wurden außerdem Zusammenhänge zwischen dem Konfliktfragebogen und weiteren klinisch relevanten Verfahren (OPDSFK, SDQ, RFQ) an der Stichprobe von 229 Patient_innen berechnet, die unter b) beschrieben sind.

\section{Messinstrumente}

\section{Befundbogen der Konfliktachse}

Der Befundbogen (Arbeitsgruppe OPD-KJ-2, 2020, S. 390) ist ein Einschätzbogen, mit dem die Therapeutin bzw. der Therapeut auf der Grundlage des OPD-KJ-Interviews sieben Konflikte der Patientin bzw. des Patienten einschätzen kann (Nähe vs. Distanz, Unterwerfung vs Kontrolle, Versorgungskonflikt, Selbstwertkonflikt, Schuldkonflikt, ödipaler Konflikt, Identitätskonflikt). Sie sind nach der Häufigkeit zu raten von $0=$ nicht vorhanden, über $1=$ vorhanden und wenig bedeutsam bis $3=$ vorhanden und sehr bedeutsam. Des Weiteren ist pro Konflikt der Verarbeitungsmodus (eher aktiv, eher passiv) anzugeben. Bei der Einschätzung kann die Operationalisierung im Manual (Arbeitsgruppe OPD-KJ-2, 2020, S. 139-196), bezogen auf die Altersstufe, den betroffenen Lebensbereich und den jeweiligen Verarbeitungsmodus, zur Hilfe genommen werden. Geratet werden immer alle Konflikte und wenn man besonders viele Indikatoren für einen bestimmten Konflikt hat, gilt dieser als „sehr bedeutsam“. Am Ende des Befundbogens ist anzugeben, welches der wichtigste und welches der zweitwichtigste Konflikt war. Außerdem sind aktuelle schwere Belastungen (in den 6 Monaten vor dem Interview) und schwere Belastungen davor anzugeben.

\section{Konfliktfragebogen}

Der Fragebogen kann von jugendlichen Patient_innen ab dem Alter von 13 Jahren (Altersstufe 3) und ihren behandelnden Psychiater_innen oder Psychotherapeut_innen ausgefüllt werden. Der Konfliktfragebogen besteht aus 28 Items, die von Patient_innen und Therapeut_innen unabhängig voneinander beantwortet werden. Dabei wird jeder der sieben intrapsychischen Konflikte nach OPD-KJ-2 mit jeweils vier Items erfasst. Die beiden vorherrschenden Verarbeitungsmodi (aktiv vs. passiv) werden dabei gleichwertig mit jeweils zwei Items abgefragt. Der Konfliktfragebogen liegt in einer Therapeuten- und einer Patientenversion vor. Zusätzlich wurde ein Konfliktgesamtwert bestimmt, um das generelle Konfliktniveau einzuschätzen. Er soll als Indikator für den Grad der Einschränkung des Alltagslebens auf Basis der intrapsychischen Konflikte dienen. Beispielitems finden sich in Tabelle 1. Das Antwortformat ist fünfstufig: $0=$ nein, $1=$ eher nein, $2=$ teils $/$ teils, $3=$ eher $j a$, $4=j a$. Der Fragebogen ist für Patient_innen der Altersstufe 3 (13.-18. Lebensjahr) vorgesehen. Die Interrater-Übereinstimmung ist signifikant $($ ICC $=.855, p<.001)$.

\section{Strukturniveau}

Wir verwendeten die Kurzversion des Strukturfragebogens von Ehrenthal et al. (OPD-SFK; Ehrenthal et al., 2015) für Erwachsene; eines der 12 Items wurde für die Jugendlichen angepasst. Der OPD-SFK wurde sowohl von den Jugendlichen als auch von den Therapeut_innen ausgefüllt. Die Itemcodierung der zugrunde liegenden Version sowie die Skalenstruktur blieben dabei erhalten. Die fünfstufige Antwortskala reicht von $0=$ trifft gar nicht $z u$ über $2=$ teils/ teils bis $4=$ trifft genau zu. Cronbachs $\alpha$ für den Gesamtmittelwert liegt bei .89 . 


\section{Mentalisierung}

Der Reflective Functioning Questionnaire (RFQ; Fonagy et al, 2016) ist ein Verfahren, das im Selbstbericht der Patien_innen die Mentalisierungsfähigkeit abfragt. Die fünfstufige Antwortskala reicht von $0=$ trifft gar nicht $z u$ über $2=$ teils/teils bis $4=$ trifft genau $z u$. Wir nutzen den Gesamtmittelwert. Zur Validität und Reliabilität vgl. Kornhas, Schröder-Pfeifer, Georg, Zettl und Taubner (2020).

\section{Patientensicht auf Beeinträchtigungen}

Der Strengths and Difficulties Questionnaire (SDQ) ist ein kurzer Fragebogen zu Verhaltensauffälligkeiten und -stärken bei Kindern und Jugendlichen, der im Fremdbericht sowie als Selbstbericht vorliegt und von Woerner et al. (2002) validiert und normiert wurde. Bei der Formulierung der 25 SDQ-Items wurde gezielt auf eine Ausgewogenheit von positiven und negativen Verhaltensaspekten geachtet. Wir benutzen die Selbstberichtsform für die „Difficulties“; für die vorliegende Auswertung bezogen wir uns auf die drei Skalen Emotionale Probleme, Externalisierende Verhaltensauffälligkeiten und Hyperaktivitätsund Aufmerksamkeitsprobleme. Die fünfstufige Antwortskala reicht von $0=$ trifft gar nicht $z u$ über $2=$ teils/teils bis 4 = trifft genau $z u$. Die Reliabilität ist mit .60 bis .76 für die drei Skalen ausreichend.

\section{Ergebnisse}

\section{Entwicklung des multiperspektivischen Konfliktfragebogens}

\section{Erstellung des Itempools und einer Langversion sowie Faktorenanalyse der Langversion}

Zunächst wurde ein Itempool von 110 Items gebildet, der der Erstellung einer Langversion mit 42 Items diente. Die Items wurden basierend auf Expertenratings aus dem initialen Pool von 110 Items ausgewählt und repräsentieren Ausprägungen der sieben Konflikte (jeweils mit aktivem und passivem Modus). Die Aussagen sollten als typisch für jemanden gelten, bei dem der jeweilige Konflikt vorhanden ist. Die Items wurden generiert, indem die konfliktbezogenen Passagen des OPD-KJ-Manuals in für jeden Konfliktbewältigungsmodus möglichst typische Aussagen für Patient_innen überführt wurden; sie sollten Manifestationen der sieben Konflikte (mit jeweils passivem und aktivem Bewältigungsmodus) repräsentieren. Anschließend wurden neun erfahrene OPD-KJ-Expert innen aus dem Arbeitskreis gebeten, die 42 Items daraufhin zu beurteilen, ob sie typisch für jemanden sind, bei dem der entsprechende Konflikt mit dem entsprechenden Verarbeitungsmodus vorliegt. Zur Beantwortung der
Frage, wie gut das Item den jeweiligen Konfliktbewältigungsmodus abbildet, wurde eine vierstufige Skala von $0=$ gar nicht bis $4=$ seh $r$ gut verwendet. Tabelle 1 gibt die Einschätzung der neun Expert_innen bzgl. der 42-Itemversion wieder.

\section{Die Erstellung der Kurzversion}

Auf der Basis der Beantwortung der 42-Itemversion durch 121 jugendliche Patient_innen und ihre 46 Therapeut_innen wurde eine Faktorenanalyse getrennt für die beiden Informanten berechnet. Die Analyse der Faktorenstruktur der 14 Skalen des Konfliktfragebogens (sieben Konflikte über je zwei Verarbeitungsmodi) mittels einer exploratorischen Faktorenanalyse (EFA) mit Maximum-Likelihood-Schätzverfahren erbrachte sowohl für die Patientendaten als auch für die Therapeutendaten eine große Anzahl der zu extrahierenden Faktoren, was auch der Screeplot der Eigenwerte mit einer späten endgültigen Verflachung bestätigte.

Für die Daten der Therapeut_innen wurden empirisch zehn Faktoren extrahiert, für die Jugendlichen neun Faktoren. Die Screeplots der Eigenwerte für die Therapeuten- und die Jugendlichenversion sind im elektronischen Supplement (ESM) 1 einzusehen. Die Varianzaufklärung der Therapeutenversion betrug bei zehn Faktoren insgesamt $76.22 \%$ und bei der Jugendlichenversion mit neun Faktoren ebenfalls lediglich $74.98 \%$ Gesamtvarianzaufklärung trotz der großen Anzahl an Faktoren. Die Varianzaufklärung war in beiden Versionen nicht gleichmäßig über die Faktoren verteilt. So trugen die ersten drei Faktoren in beiden Versionen bereits die Hälfte zur gesamten Varianzaufklärung bei (Therapeutenversion 38.94\%; Jugendlichenversion 35.66\%). Der Abfall der Varianzaufklärung wird auch im Screeplot der Eigenwerte (vgl. ESM 1) deutlich. Da eine 3-Faktoren-Lösung allerdings nicht den inhaltlich notwendigen Differenzierungsgrad widerspiegelt und die weiteren Faktoren nur wenig zur weiteren Varianzaufklärung beitrugen, entschieden wir uns gegen ein faktorenanalytisch geleitetes Vorgehen.

Wir entschlossen uns daher, für die Kurzversion der OPD-KF diejenigen Items auszuwählen, die die jeweils theoretisch formulierten, intrapsychischen Konfliktthemen am ehesten abbildeten. Hierzu wurden zwei Kriterien verwendet: Erstens sollte die Mehrheit der Expert_innen (d.h. mindestens sechs von neun) zu dem Schluss kommen, dass das Item den Konflikt mindestens „befriedigend“ abbildet (d.h. mit 3 oder 4 beurteilen); zweitens wählten wir anhand der Beurteilerübereinstimmung die Items aus, die in der Skala eine ICC $>.80$ aufwiesen. Die Items und Skalen der Langversion sind in Tabelle 1 dargestellt. Die Beurteilerübereinstimmungen ICC pro Skala sind jeweils für die Langversion $\left(\mathrm{ICC}_{\text {lang }}\right)$, d.h. für die sechs Items, und für die reduzierte Kurzversion $\left(\mathrm{ICC}_{\mathrm{kurz}}\right)$, d.h. für die vier fettgedruckten 
Items, aufgeführt. Die Items, die für die Kurzversion erhalten geblieben sind, sind fett markiert. Der Range der Beurteilerübereinstimmung für die sieben Skalen lag zwischen .21 und .47, d.h., die Items der Langversion repräsentierten den jeweiligen Konflikt noch nicht optimal. Nach Reduktion der Items auf vier Items pro Skala, insgesamt 28 Items, erfüllten die meisten Skalen das Kriterium einer befriedigenden bis sehr guten Beurteilerübereinstimmung. So steigt die Beurteilerübereinstimmung durch die Itemreduktion von sechs auf vier Items pro Skala in allen Skalen an: Nähe-Distanz (.278 auf .731), Unterwerfung vs. Kontrolle
(.353 auf .680), Versorgung (.083 auf .829), Selbstwert (.012 auf .258), Schuldkonflikt (.340 auf .791), ödipaler Konflikt (.324 auf .454) und Identitätskonflikt (.469 auf .564). Die Werte sind mit ihrem Signifikanzniveau jeweils am Ende der Skala in Tabelle 1 abgetragen.

Sodann wurde eine „Ich“-Form für die Selbsteinschätzung der Jugendlichen und eine „er/sie“-Form für die Therapeutin bzw. den Therapeuten erstellt und der Antwortmodus mit $0=$ nein $=$ trifft gar nicht $z u, 1=$ eher nein $=$ trifft eher nicht zu, 2 = teils/teils = trifft teilweise $z u, 3=$ eher ja $=$ trifft eher $z u, 4=j a=$ trifft genau $z u$ festgelegt.

Tabelle 1. Einschätzungen der neun Expert_innen über die 42 Items der Konfliktskalen.

\begin{tabular}{|c|c|c|c|c|c|c|c|}
\hline & & & \multicolumn{5}{|c|}{ Repräsentiert den Konflikt: } \\
\hline \multicolumn{3}{|c|}{ Items } & $\begin{array}{l}0=\text { gar } \\
\text { nicht }\end{array}$ & $\begin{array}{l}1=\text { eher } \\
\text { nicht }\end{array}$ & $\begin{array}{l}2=\text { teils } \\
\text { teils }\end{array}$ & $\begin{array}{l}3= \\
\text { gut }\end{array}$ & $\begin{array}{l}4=\text { sehr } \\
\text { gut }\end{array}$ \\
\hline \multirow{6}{*}{ 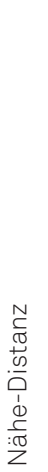 } & 1. & $\mathrm{Er} /$ sie ist am liebsten nur mit den Eltern zusammen. & 0 & 1 & 2 & 5 & 1 \\
\hline & 2. & Die Beziehung zu seinen/ihren Eltern ist ihr/ihm definitiv zu eng. & 0 & 0 & 0 & 3 & 6 \\
\hline & 3. & Er/sie verliert in Beziehungen schnell das Interesse an anderen. & 0 & 2 & 3 & 4 & 0 \\
\hline & 4. & $\begin{array}{l}\text { Kooperation und Gruppenarbeiten fallen ihm/ihr schwer, sie/er arbeitet } \\
\text { am liebsten alleine. }\end{array}$ & 0 & 1 & 2 & 4 & 2 \\
\hline & 5. & $\begin{array}{l}\text { In engen Freundschaften/Beziehungen hat sie/er ständig Angst vor Ver- } \\
\text { lust. }\end{array}$ & 0 & 0 & 0 & 2 & 7 \\
\hline & $\begin{array}{l}6 . \\
\text { ICC }\end{array}$ & $\begin{array}{l}\text { Sie/er kann sich schlecht von den Eltern trennen. } \\
\text { ang): .278, } p=.003 ; \text { ICC (kurz): .731, } p<.001\end{array}$ & 0 & 0 & 1 & 2 & 6 \\
\hline \multirow{6}{*}{ 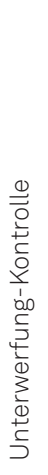 } & 7. & In allen Beziehungen ordnet sie/er sich auffallend gerne unter. & 0 & 0 & 0 & 2 & 7 \\
\hline & 8. & $\begin{array}{l}\text { Er/sie kümmert sich nicht um Regeln, egal ob in der Schule, auf Station, } \\
\text { zu Hause. }\end{array}$ & 0 & 0 & 4 & 2 & 3 \\
\hline & 9. & $\begin{array}{l}\text { Die Spielregeln in seiner/ihrer Familie sind so eng, dass er/sie häufig } \\
\text { dagegen verstößt. }\end{array}$ & 1 & 0 & 5 & 2 & 1 \\
\hline & 10. & Unter den Freunden hat er/sie eindeutig das Sagen. & 0 & 0 & 2 & 4 & 3 \\
\hline & 11. & In nahen Beziehungen übt er/sie gerne Macht und Kontrolle aus. & 0 & 0 & 0 & 2 & 7 \\
\hline & $\begin{array}{ll}12 . \\
\text { ICC }\end{array}$ & $\begin{array}{l}\text { Er/sie rebelliert nicht offiziell, macht aber dann doch was er/sie möchte. } \\
\text { ang): .353, } p<.001 ; \text { ICC (kurz): .680, } p=.045\end{array}$ & 0 & 0 & 1 & 5 & 3 \\
\hline \multirow{6}{*}{ 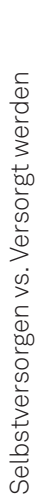 } & 13. & $\begin{array}{l}\text { Er/sie wird von den Eltern regelrecht überschüttet mit allem, was er/sie } \\
\text { braucht. }\end{array}$ & 1 & 1 & 2 & 2 & 3 \\
\hline & 14. & $\begin{array}{l}\text { Um die Bedürfnisse des Patienten/der Patientin kümmert sich wirklich } \\
\text { niemand in der Familie. }\end{array}$ & 0 & 1 & 1 & 4 & 3 \\
\hline & 15. & Er/sie muss häufig für sich selbst sorgen/auf sich selbst achten. & 0 & 0 & 0 & 3 & 6 \\
\hline & 16. & Er/sie kommt in Beziehungen zu Gleichaltrigen oft zu kurz. & 0 & 0 & 4 & 1 & 4 \\
\hline & 17. & $\begin{array}{l}\text { Er/sie achtet peinlich genau darauf, dass die Bedürfnisse anderer ausrei- } \\
\text { chend bedacht werden. }\end{array}$ & 0 & 0 & 3 & 3 & 3 \\
\hline & 18. & $\begin{array}{l}\text { Ihm/ihr ist es wichtig, dass andere sich nur um ihn/sie kümmern. } \\
\text { Ing): .083, } p=.263 ; \text { ICC (kurz): .829, } p=.017\end{array}$ & 0 & 0 & 2 & 3 & 4 \\
\hline
\end{tabular}




\begin{tabular}{|c|c|c|c|c|c|c|c|}
\hline & 19. & Er/sie will ständig bewundert werden. & 0 & 0 & 0 & 2 & 7 \\
\hline \multirow{6}{*}{ 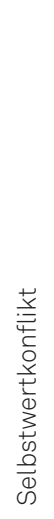 } & 20. & $\begin{array}{l}\text { Es ist ihm/ihr wichtig, bessere Leistungen zu erbringen als andere, da- } \\
\text { mit man sieht, dass er/sie allen überlegen ist. }\end{array}$ & 0 & 0 & 1 & 3 & 5 \\
\hline & 21. & $\begin{array}{l}\text { Er/sie glaubt, es nicht verdient zu haben, von anderen wertgeschätzt zu } \\
\text { werden. }\end{array}$ & 0 & 0 & 1 & 2 & 6 \\
\hline & 22. & $\begin{array}{l}\text { Er/sie vergleicht sich sehr häufig mit Mitschülern/Mitschülerinnen und } \\
\text { das geht meistens negativ aus. }\end{array}$ & 0 & 1 & 0 & 2 & 6 \\
\hline & 23. & Bestätigung durch andere ist inm/ihr das Wichtigste. & 0 & 0 & 1 & 2 & 6 \\
\hline & 24. & $\begin{array}{l}\text { Er/sie schämt sich, dass er/sie nicht so kompetent und attraktiv ist wie } \\
\text { andere. }\end{array}$ & 0 & 1 & 0 & 5 & 3 \\
\hline & ICC & ang): .012, $p=.379 ;$ ICC (kurz): .258, $p<.017$ & & & & & \\
\hline \multirow{7}{*}{ 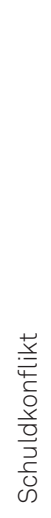 } & 25. & Er/sie macht sich Vorwürfe, wenn etwas in der Familie schiefgeht. & 0 & 0 & 0 & 2 & 7 \\
\hline & 26. & $\begin{array}{l}\text { Er/sie fühlt sich dafür verantwortlich, dass es allen in der Familie gut } \\
\text { geht. }\end{array}$ & 0 & 1 & 0 & 3 & 5 \\
\hline & 27. & $\mathrm{Er} /$ sie fürchtet, er/sie habe sich nicht genug um Mutter/Vater gekümmert. & 0 & 1 & 0 & 3 & 5 \\
\hline & 28. & Aus seiner/ihrer Sicht, hat er/sie die schrecklichsten Eltern der Welt. & 0 & 1 & 4 & 2 & 2 \\
\hline & 29. & Ihm/ihr ist es egal, wie es Mutter/Vater geht. Das ist deren Sache. & 1 & 0 & 5 & 3 & 0 \\
\hline & 30. & $\begin{array}{l}\text { Er/sie beschuldigt seine/ihre Eltern, dass es ihm/ihr heute so schlecht } \\
\text { geht. }\end{array}$ & 0 & 0 & 2 & 5 & 2 \\
\hline & ICC & ang): .340, $p<.001 ;$ ICC (kurz): .791, $p=.011$ & & & & & \\
\hline \multirow{7}{*}{ 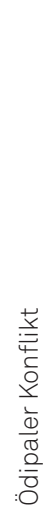 } & 31. & $\begin{array}{l}\text { Er/sie konkurriert oft um den attraktivsten Jungen/das attraktivste } \\
\text { Mädchen. }\end{array}$ & 0 & 0 & 0 & 3 & 6 \\
\hline & 32. & Gruppen, in denen es nicht um Sexualität geht, langweilen ihn/sie. & 0 & 0 & 4 & 5 & 0 \\
\hline & 33. & $\mathrm{Er} /$ sie beeindruckt gerne andere mit seinem/ihrem Körper. & 0 & 0 & 1 & 1 & 7 \\
\hline & 34. & Im Umgang mit dem anderen Geschlecht ist er/sie äußerst gehemmt. & 0 & 0 & 1 & 1 & 7 \\
\hline & 35. & Er/sie interessiert sich noch nicht für Sexualität/das andere Geschlecht. & 0 & 0 & 0 & 6 & 3 \\
\hline & 36. & $\begin{array}{l}\text { Er/sie ist genervt darüber, dass Sexualität überall in den Medien so viel } \\
\text { thematisiert wird. }\end{array}$ & 0 & 1 & 0 & 2 & 6 \\
\hline & ICC & ang): .324, $p<.001 ;$ ICC (kurz): .454, $p<.001$ & & & & & \\
\hline \multirow{7}{*}{ 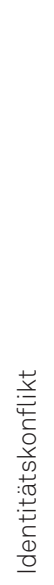 } & 37. & Er/sie ist ständig auf der Suche nach neuen Identitätsentwürfen. & 0 & 0 & 0 & 6 & 3 \\
\hline & 38. & $\begin{array}{l}\text { Er/sie kann sich nicht festlegen, wer er/sie sein möchte, die vielen Mög- } \\
\text { lichkeiten machen zu viel Angst. }\end{array}$ & 0 & 0 & 1 & 5 & 3 \\
\hline & 39. & Er/sie hat ständig neue Idole, denen er/sie unkritisch nacheifert. & 0 & 0 & 0 & 3 & 6 \\
\hline & 40. & $\begin{array}{l}\text { Er/sie scheint getrieben, ständig Neues (äußere Erscheinung, Hobbies, } \\
\text { Beziehungen) auszuprobieren. }\end{array}$ & 0 & 0 & 2 & 1 & 6 \\
\hline & 41. & $\begin{array}{l}\text { Am liebsten würde er/sie so bleiben, wie er/sie ist, jede Veränderung kann } \\
\text { doch schlimme Folgen haben. }\end{array}$ & 0 & 1 & 6 & 2 & 0 \\
\hline & 42. & $\begin{array}{l}\text { Er/sie hat keine Ahnung, wer er/sie ist und wie er/sie das herausfinden } \\
\text { kann. }\end{array}$ & 0 & 0 & 0 & 3 & 6 \\
\hline & \multicolumn{7}{|c|}{ ICC (lang): .469, $p<.001 ;$ ICC (kurz): .564, $p<.001$} \\
\hline
\end{tabular}




\section{Kriteriumsvalidität: Vergleich zwischen einer Stichprobe von ambulanten Psychotherapiepatient_ innen und einer altersgleichen unauffälligen Stichprobe und die Standardisierung durch Erstellung von $T$-Werten}

Im nächsten Schritt wurden an einer Stichprobe von insgesamt $N=340$ Jugendlichen aus den Studien a) und b) und 46 Therapeut_innen aus Studie a) die Daten im Konfliktfragebogen, basierend auf der Kurzversion von 28 Items, analysiert und $T$-Werte erstellt.

Datengrundlage für den Vergleich zwischen Patient innen und der Kontrollstichprobe waren 229 ambulante Patient_innen und 198 gesunde, altersgleiche Jugendliche. Tabelle 2 gibt die entsprechenden Kennwerte für die Patientenstichprobe und die Kontrollstichprobe wieder, bezogen auf den Gesamtmittelwert Konflikte und den Mittelwert pro Konflikt, über den aktiven und passiven Verarbeitungsmodus summiert.

\section{Unterschiede zwischen Patient_innen und Gesunden} Anschließend wurden univariate Varianzanalysen (ANOVAs) mit dem Gesundheitsstatus als unabhängiger Variable (Gesunde vs. Patient_innen) und den Konfliktskalen berechnet. Die ANOVAs ergeben signifikante Unterschiede zwischen den beiden Gruppen. Die Patient innen geben signifikant höhere Werte in allen Konfliktskalen und dem Gesamtwert an: Der deutlichste Effekt zeigt sich im Konfliktgesamtwert $\left(F[1,421]=57.553, p<.001, \eta^{2}=.119\right)$. Innerhalb der Konfliktskalen zeigt sich der größte Unterschied im Schuldkonflikt $(F[1,421]=40.742, p<$ $\left..001, \eta^{2}=.088\right)$ und beim Konflikt Selbst versorgen vs. Versorgtwerden $\left(F[1,421]=33.147, p<.001, \eta^{2}=.072\right)$. In den anderen Konflikten ergeben sich vergleichbare, geringere Effekte: Nähe-Distanz $(F[1,421]=14.242, p<.001$, $\left.\eta^{2}=.032\right)$, Unterwerfung vs. Kontrolle $(F[1,421]=12.441$, $\left.p<.001, \eta^{2}=.028\right)$, Selbstwertkonflikt $(F[1,421]=12.633$, $\left.p<.001, \eta^{2}=.029\right)$ und Identitätskonflikt $(F[1,421]=15.827$, $\left.p<.001, \eta^{2}=.036\right)$. Im ödipalen Konflikt ergibt sich die geringste Effektstärke $(F[1,421]=4.183, p=.041$, $\left.\eta^{2}=.010\right)$.

\section{Standardisierung anhand von $T$-Werten}

Die Umrechnung in $T$-Werte für alle 14 Skalen des Konfliktfragebogens (sieben Konflikte jeweils mit aktivem und passivem Verarbeitungsmodus), bezogen auf die Selbstund die Therapeuteneinschätzung, findet sich im ESM 2. Die Standardisierung bezog sich auf die Daten der 340 ambulanten Patient_innen aus den Studien a) und b) und auf die Therapeuteneinschätzung der 46 Therapeut_innen aus Studie a). In der Tabelle sind die Rohwerte für die Mittelwerte der $T$-Verteilung (50) sowie die Rohwerte eine

Tabelle 2. Deskriptive Statistiken der intrapsychischen Konflikte in der Patientenstichprobe $(N=229)$ und der Kontrollstichprobe ( $N=198)$

\begin{tabular}{|c|c|c|c|c|c|c|c|c|c|c|c|c|c|c|}
\hline & \multicolumn{7}{|c|}{ Patienten } & \multicolumn{7}{|c|}{ Kontrollstichprobe } \\
\hline & $M$ & $S D$ & $S E_{M}$ & Min & Max & Schiefe & Kurtosis & $M$ & $S D$ & $S E_{\mathrm{M}}$ & Min & Max & Schiefe & Kurtosis \\
\hline $\begin{array}{l}\text { Gesamt- } \\
\text { konfliktwert }\end{array}$ & 1.35 & .33 & .02 & .43 & 2.32 & -.25 & .22 & 1.11 & .32 & .02 & .21 & 2.36 & .53 & 1.73 \\
\hline $\begin{array}{l}\text { Nähe vs. } \\
\text { Distanz }\end{array}$ & 1.39 & .64 & .04 & .00 & 3.50 & .09 & -.27 & 1.16 & .59 & .04 & .00 & 2.75 & .37 & -.32 \\
\hline $\begin{array}{l}\text { Unterwerfung } \\
\text { vs. Kontrolle }\end{array}$ & 1.38 & .55 & .04 & .00 & 3.25 & .29 & .47 & 1.20 & .50 & .03 & .00 & 2.50 & -.02 & -.58 \\
\hline $\begin{array}{l}\text { Selbst versor- } \\
\text { gen vs. Ver- } \\
\text { sorgtwerden }\end{array}$ & 1.60 & .53 & .04 & .25 & 3.25 & .05 & -.33 & 1.32 & .46 & .03 & .00 & 2.50 & -.11 & .16 \\
\hline $\begin{array}{l}\text { Selbstwert- } \\
\text { konflikt }\end{array}$ & 1.44 & .69 & .05 & .00 & 4.00 & .26 & .49 & 1.20 & .65 & .04 & .00 & 3.75 & .68 & .75 \\
\hline $\begin{array}{l}\text { Schuld- } \\
\text { konflikt }\end{array}$ & 1.27 & .67 & .04 & .00 & 3.50 & .25 & -.34 & .89 & .52 & .03 & .00 & 2.75 & .08 & -.25 \\
\hline $\begin{array}{l}\text { Ödipaler } \\
\text { Konflikt }\end{array}$ & 1.16 & .65 & .04 & .00 & 2.75 & .21 & -.68 & 1.04 & .56 & .03 & .00 & 2.50 & .07 & -.44 \\
\hline $\begin{array}{l}\text { Identitäts- } \\
\text { konflikt }\end{array}$ & 1.21 & .68 & .04 & .00 & 3.25 & .07 & -.50 & .96 & .63 & .04 & .00 & 3.50 & .62 & .81 \\
\hline
\end{tabular}


Standardabweichung unter dem Mittelwert (40) und eine Standardabweichung über dem Mittelwert (60) für zukünftige Referenz angegeben.

\section{Konstruktvalidität: Vergleich von Operationalisierung durch Befundbogen (Arbeitsgruppe OPD-KJ-2, 2020) mit dem Konfliktfragebogen für Therapeut_innen}

Im nächsten Schritt fokussierten wir stärker auf der Fremdeinschätzung durch Therapeut_innen und verglichen zwei verschiedene Erhebungsmethoden miteinander, die Operationalisierung durch den Befundbogen im Buch (Arbeitsgruppe OPD-KJ-2, 2020, S. 390) und die Operationalisierung in den Konfliktskalen für Therapeut_ innen. Einbezogen wurden zwei Trainingsstichproben von insgesamt $N=52$ Therapeut_innen sowie die Einschätzung des gleichen klinischen Falles durch drei Expert_innen.

Zunächst wurde wie beschrieben ein Video einer stationär aufgenommenen Jugendlichen mit einer F92-Diagnose ausgewählt, die von einem männlichen Therapeuten interviewt wurde. Drei erfahrene OPD-KJ-Trainer_innen schätzten die Patientin anhand des Konfliktfragebogens ein. Es ergaben sich Interraterreliabilitäten bezogen auf die Konflikte von .582 bis .976 . Der Konfliktgesamtwert hatte eine ICC von $.861(p<.001)$.

Das Video dieser Patientin wurde dann im Rahmen eines OPD-KJ-Trainings den Teilnehmer_innen vorgeführt. Es handelte sich um zwei Trainingsgruppen mit insgesamt 52 Teilnehmer_innen. Die Berechnung der ICCs benötigt Varianz zwischen den einzelnen Items. Da in der Erhebung mit dem Befundbogen keine einzelnen Items beantwortet werden, sondern direkt die Werte pro Konflikt und Verarbeitungsmodus (Versorgungskonflikt aktiver Modus, Versorgungskonflikt passiver Modus etc.) eingeschätzt werden, konnte nur eine ICC für den Konfliktgesamtwert gebildet werden (ICC $=.972 ; p<.001$ ). Die Erhebung anhand der Konfliktskalen ergab hohe Interraterreliabilitäten: Der Konfliktgesamtwert hatte eine ICC von .825 $p<$ .001). Die Skalen hatten ebenfalls hohe ICCs: Nähe vs. Distanz (ICC $=.913 ; p<.001)$, Unterwerfung vs. Kontrolle (ICC $=.748 ; p=.009)$, Selbst versorgen vs. Versorgt werden $($ ICC $=.984 ; p<.001)$, Selbstwertkonflikt $($ ICC $=.884$; $p<.001$ ), Schuldkonflikt (ICC $=.986 ; p<.001$ ), ödipaler Konflikt (ICC $=.918 ; p<.001)$ und Identitätskonflikt (ICC $=.793 ; p=.005)$.

Die 52 Einschätzungen nach dem ausführlichen Interviewleitfaden aus dem Buch auf Basis des Befundbogens (Arbeitsgruppe OPD-KJ-2, 2020, S. 390) auf der einen Seite und mit den Konfliktskalen auf der anderen Seite wurden anschließend anhand von $t$-Tests für abhängige Stichproben verglichen. In den $t$-Tests für abhängige
Stichproben zeigt sich, dass die Einschätzungen der Konflikte, differenziert nach aktivem und passivem Verarbeitungsmodus, nicht signifikant voneinander abweichen. Allerdings zeigt eine Überprüfung auf der Ebene des Konfliktgesamtwertes einen signifikant höheren Wert der Einschätzung mit dem Befundbogen $(M=33.69$; $S D=2.35)$ im Vergleich zur Einschätzung mit den Konfliktskalen $(M=29.46 ; S D=5.79 ; p=.003, T[51]=-3.117)$. Abbildung 1 veranschaulicht den Vergleich der beiden Erhebungsmethoden.

\section{Diskriminante Validität: Zusammenhänge zwischen den Konfliktskalen und Maßen der Symptombelastung, des Strukturniveaus und der Mentalisierung}

Im letzten Schritt wurden Zusammenhänge zwischen den Konfliktfragebögen für Patient_innen, dem Strukturgesamtwert, der Mentalisierung (Reflective Functioning Questionnaire) und der Symptombelastung (Mittelwert der Skalen Emotionale Probleme, Verhaltensauffälligkeiten und Hyperaktivität des Strengths and Difficulties Questionnaire) ermittelt. Datengrundlage waren 230 Patient_innen.

Die Struktur korreliert signifikant positiv mit Symptombelastung $(r=.189 ; p=.004)$ und Mentalisierung $(r=.280$; $p<.001)$. Dies bedeutet, dass höhere Strukturdefizite mit höherer Symptombelastung und niedrigerer Mentalisierungsfähigkeit assoziiert sind. Die Konfliktskalen und der Konfliktgesamtwert korrelieren nicht mit der Struktur, der Mentalisierung und der Symptombelastung. Die Ergebnisse der Korrelationen sind in Tabelle 3 ausführlich dargestellt.

\section{Diskussion}

Fragebogenversionen zur Erfassung der Bewältigungsmodi unbewusster Konflikte gemäß der Operationalisierten Psychodynamischen Diagnostik (OPD-KJ) liegen bislang nur von der Arbeitsgruppe um Cord Benecke (Hold et al., 2015) vor, zentrieren aber auf die narzisstische Selbsteinschätzung. Der hier entwickelte OPD-KJ-Konfliktfragebogen legt eine Version für Patient_innen und ihre Therapeut_innen vor. Der Fragebogen umfasst in seiner Endversion 28 Items und kann von jugendlichen Patient innen und ihren behandelnden Psychiater innen oder Psychotherapeut innen ausgefüllt werden. Dabei wird jeder intrapsychische Konflikt nach OPD-KJ-2 mit jeweils vier Items (für die Verarbeitungsmodi aktiv vs. passiv) erfasst. Diese Kurzversion wurde in der Selbst- und Fremdeinschätzung in einem vierstufigen Verfahren erstellt, an dem 


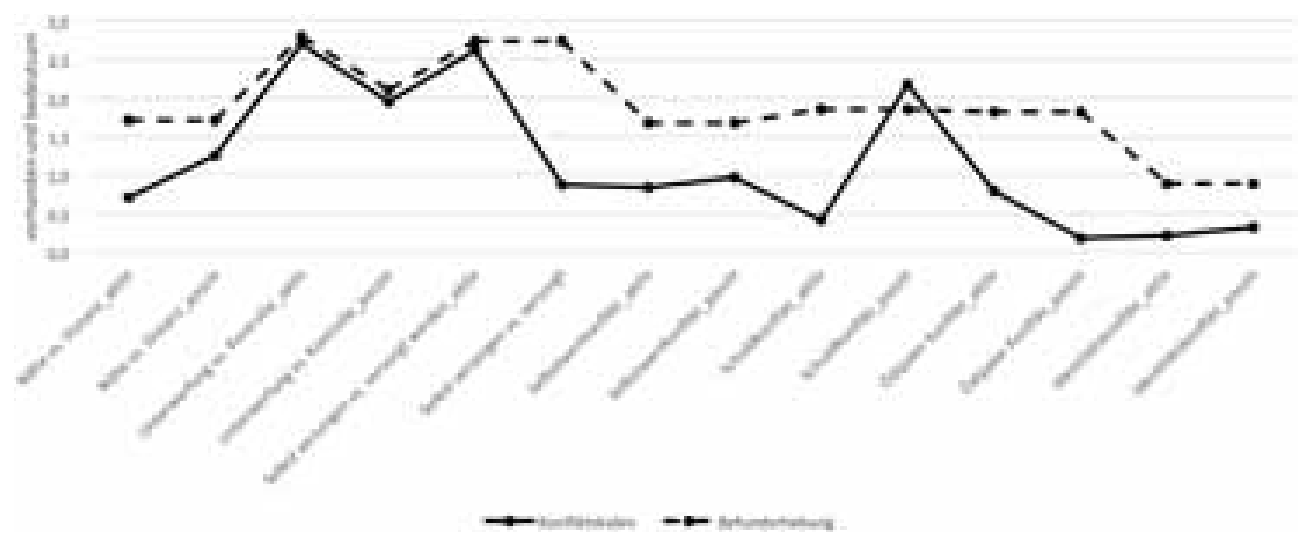

Abbildung 1. Vergleich der Einschätzungen der intrapsychischen Konflikte nach OPD-KJ anhand der Befunderhebung und der Konfliktskalen $(N=52)$ an einem videografierten Einzelfall. ${ }^{3}$

Anmerkung: Die Skalierung der y-Achse ist 0 = nicht vorhanden, 1 = vorhanden und wenig bedeutsam, $2=$ vorhanden und bedeutsam, $3=$ vorhanden und sehr bedeutsam.

Tabelle 3. Pearson-Korrelationen zwischen dem Gesamtkonfliktniveau, den Konfliktskalen, der Symptombelastung, der Struktur und Mentalisierung $(N=229)$.

\begin{tabular}{|c|c|c|c|c|c|c|c|c|c|c|c|}
\hline Skalen & & 2 & 3 & 4 & 5 & 6 & 7 & 8 & 9 & 10 & 11 \\
\hline \multirow[t]{2}{*}{ Gesamtkonfliktwert (1) } & r & $.60 * \star$ & $.47 * \star$ & $.41 * \star$ & $.63^{* *}$ & $.60 * *$ & $.54 \star \star$ & $.67 * \star$ & .05 & .07 & .12 \\
\hline & $p$ & $<.001$ & $<.001$ & $<.001$ & $<.001$ & $<.001$ & $<.001$ & $<.001$ & .422 & .313 & .059 \\
\hline \multirow[t]{2}{*}{ Nähe vs. Distanz (2) } & r & & $.20 * *$ & .089 & $.28 * \star$ & $.26 * \star$ & $.21 * \star$ & $.29 * \star$ & .055 & -.042 & .056 \\
\hline & $p$ & & $<.001$ & .065 & $<.001$ & $<.001$ & $<.001$ & $<.001$ & .404 & .529 & .401 \\
\hline \multirow[t]{2}{*}{ Unterwerfung vs. Kontrolle (3) } & r & & & $.15^{\star \star}$ & $.15^{\star \star}$ & .092 & $.16 * \star$ & $.20 * \star$ & .068 & .116 & .053 \\
\hline & $p$ & & & $<.001$ & .003 & .058 & $<.001$ & $<.001$ & .302 & .079 & .426 \\
\hline \multirow[t]{2}{*}{ Selbst versorgen vs. Versorgtwerden (4) } & r & & & & $.13^{* *}$ & $.19 * *$ & .073 & $.11^{\star}$ & -.043 & .019 & .099 \\
\hline & $p$ & & & & .006 & $<.001$ & .130 & .023 & .512 & .780 & .135 \\
\hline \multirow[t]{2}{*}{ Selbstwertkonflikt (5) } & r & & & & & $.25 * \star$ & $.17 * \star$ & $.38 * \star$ & .035 & -.026 & .075 \\
\hline & $p$ & & & & & $<.001$ & $<.001$ & $<.001$ & .602 & .700 & .258 \\
\hline \multirow[t]{2}{*}{ Schuldkonflikt (6) } & r & & & & & & $.23 * \star$ & $.29 * \star$ & -.002 & .065 & .078 \\
\hline & $p$ & & & & & & $<.001$ & $<.001$ & .971 & .326 & .242 \\
\hline \multirow[t]{2}{*}{ Ödipaler Konflikt (7) } & r & & & & & & & $.26 \star \star$ & -.009 & .102 & .025 \\
\hline & $p$ & & & & & & & $<.001$ & .895 & .121 & .708 \\
\hline \multirow[t]{2}{*}{ Identitätskonflikt (8) } & r & & & & & & & & .095 & .022 & .074 \\
\hline & $p$ & & & & & & & & .152 & .740 & .263 \\
\hline \multirow[t]{2}{*}{ Struktur (9) } & r & & & & & & & & & $.19 * *$ & $28 * *$ \\
\hline & $p$ & & & & & & & & & .004 & $<.001$ \\
\hline \multirow[t]{2}{*}{ Symptombelastung (10) } & r & & & & & & & & & & .118 \\
\hline & $p$ & & & & & & & & & & .074 \\
\hline \multirow[t]{2}{*}{ Mentalisierung (11) } & r & & & & & & & & & & \\
\hline & $p$ & & & & & & & & & & \\
\hline
\end{tabular}

Anmerkungen: ${ }^{*} p<.05 ;{ }^{* *} p<.01$ 
in den verschiedenen Phasen insgesamt 340 jugendliche Patient_innen, 198 altersgleiche gesunde Jugendliche, 98 Therapeut_innen und 12 Expert_innen teilnahmen.

Die Ergebnisse der Faktorenanalyse waren wenig zufriedenstellend und ergaben für die Patienten- und Therapeutenversion übereinstimmend viele Faktoren (zehn in der Therapeutenversion und neun in der Patientenversion, mit einer Varianzaufklärung von $76 \%$ bzw. 75\%) und sind damit ähnlich wie in der Studie von Hold et al. (2015), die bei einem Selbstratingverfahren für Jugendliche 14 Faktoren (Gesamtvarianzaufklärung $57 \%$ ) berichteten, oder in dem Konfliktfragebogen für Erwachsene, bei dem Benecke et al. (2018) fünf korrelierte Faktoren fanden.

Wir entschieden uns daher für ein Vorgehen, bei dem die Expertise erfahrener Trainer_innen einbezogen wurde. Für die Kurzversion der OPD-KF mit 28 Items wurden aus einer Langversion von 42 Items diejenigen Items ausgewählt, die aus Expertensicht die theoretisch vorgesehenen Konflikte gut abbildeten, d.h., wir wählten anhand der Beurteilerübereinstimmung diejenigen Items aus, die eine ICC $>.80$ hatten. Die Beurteilerübereinstimmung bei der Langversion variierte über die Konflikte zwischen .21 und .47, d.h., die Items der Langversion repräsentierten den jeweiligen Konflikt noch nicht optimal. Nach Reduktion der Skalen auf vier Items pro Skala, insgesamt 28 Items, wiesen die meisten Skalen sehr gute bis befriedigende ICCs auf; lediglich in Bezug auf den Selbstwertkonflikt sind die Ergebnisse unbefriedigend und bedürfen weiterer Forschung zur Klärung der Ursachen. Bezogen auf den Konfliktgesamtwert, der auch bei den Studien zur Interraterreliabilität auf Basis des Befundbogens in den Studien von Rathgeber et al. (2014), SeiffgeKrenke et al. (2011), Stefini et al. (2013) und Winter et al. (2011) benutzt wurde, sind die Interraterübereinstimmungen des Konfliktfragebogens in der Therapeutenversion vielversprechend. Die Interraterübereinstimmung der gleichen Patientin ergab sowohl aus der Sicht von Expert_innen (Gesamt ICC $=.861$ ) als auch aus der Sicht von Teilnehmer_innen des OPD-KJ-Trainings (Gesamt ICC $=.825$ ) hohe Übereinstimmungen bei der Einschätzung mit der Therapeutenversion der Konfliktskalen. Desgleichen konnten hohe Beurteilerübereinstimmungen erzielt werden, wenn Therapeut_innen verschiedene Patient_innen einstufen (Gesamt ICC $=.855)$. Die Ergebnisse zeigen, dass es insgesamt gelungen ist, eine gut strukturierte Fragebogenversion zu entwickeln, die aus Sicht von Therapeut innen eine sehr gute Einschätzung der relevanten intrapsychischen entwicklungsbehindernden Konflikte erlaubt.

Es ergaben sich im Übrigen keine Unterschiede in Abhängigkeit von der Methode, wenn die gleiche Patientin sowohl nach dem Befundbogen aus dem Manual (Arbeits- kreis OPD-KJ-2, 2020) als auch anhand des Konfliktfragebogens eingeschätzt wurde, was dafür spricht, dass beide Methoden gleichwertig sind. Bei beiden Methoden wurden der Patientin mit F92-Diagnose übereinstimmend vor allem hohe Werte in Unterwerfung vs. Kontrolle (aktiv) und Versorgung (aktiv) zugeschrieben. In den $t$-Tests für abhängige Stichproben wichen die Einschätzungen der Konflikte, differenziert nach aktivem und passivem Verarbeitungsmodus, in den beiden Methoden nicht signifikant voneinander ab. Allerdings zeigt eine Überprüfung auf der Ebene des Konfliktgesamtwertes einen signifikant höheren Wert der Einschätzung mit dem Befundbogen im Vergleich zur Einschätzung mit den Konfliktskalen. Auch die grafische Veranschaulichung in Abbildung 1 verdeutlicht das höhere Niveau in der Einschätzung nach Befundbogen in den meisten Konflikten, verglichen mit der Einschätzung der gleichen Patientin mit der Therapeutenversion der Konfliktskalen. Dieser interessante Befund erklärt sich möglicherweise dadurch, dass die Teilnehmer_innen zur Einschätzung des Konfliktniveaus anhand des Befundbogens die Operationalisierung im Manual verwendeten, die eine differenzierte Einschätzung jedes Konfliktes und seines Verarbeitungsmodus nach Altersgruppe und Entwicklungskontext mit den entsprechenden Ankerbeispielen beschreibt. Das ermöglicht offensichtlich eine umfassendere Sicht auf die Konflikte, was zu entsprechend höheren Werten geführt haben mag.

Unterschiede in der Höhe des Konfliktniveaus finden sich auch noch in einem anderen Ergebnis unserer Studie: Jugendliche Patient_innen geben signifikant höhere Werte in allen Konfliktskalen und dem Gesamtwert über alle Konflikte an, verglichen mit altersgleichen gesunden Jugendlichen. Die Effektstärken im Sinne Cohens (1988) waren groß beim Konfliktgesamtwert $\left(\eta^{2}=.12\right)$. Auch bzgl. des Schuldkonflikts $\left(\eta^{2}=.09\right)$ und beim Konflikt Selbst versorgen vs. Versorgtwerden $\left(\eta^{2}=.07\right)$ liegen die Effektstärken noch im mittleren Bereich, bei den übrigen Konflikten im mittleren bis kleinen Bereich, mit der geringsten Effektstärke $\left(\eta^{2}=.01\right)$ beim ödipalen Konflikt. Interessant ist nun, dass zwar signifikante Unterschiede zwischen jugendlichen Patient_innen und altersgleichen gesunden Jugendlichen über alle Konflikte bestehen, dass die Unterschiede, wenn man die Mittelwerte anschaut, aber nicht so massiv sind wie bei einer anderen Studie, in der die Konflikte mit dem Befundbogen eingeschätzt wurden. Hier zeigte sich nämlich (vgl. SeiffgeKrenke et al., 2014) in einzelnen Konflikten ein zwei- bis dreimal so hoher Wert in der Patientenstichprobe, verglichen mit der gesunden altersgleichen Kontrollgruppe. Auch dies scheint zu bestätigen, dass die beiden Erhebungsmethoden vergleichbare Ergebnisse erbringen, jedoch die Einschätzung anhand des Befundbogens insgesamt etwas höher ausfällt. 
Zur Überprüfung der diskriminanten Validität der Konfliktfragebögen wurden die Zusammenhänge mit Instrumenten zur Erfassung der Struktur (OPD-SFK) sowie der Mentalisierung (RFQ) und der Symptombelastung (SDQ) ermittelt. Zwar ergaben sich Zusammenhänge mit dem Strukturfragebogen, jedoch keine mit den Konfliktskalen. Diese Ergebnisse sind auf den ersten Blick kontraintuitiv und wurden von uns so nicht erwartet. In Folgeuntersuchungen werden wir darauf eingehen, ob dies methodische oder inhaltliche Gründe hat. Inhaltlich ist es möglich, dass Konflikte weniger abhängig von der Mentalisierungsfähigkeit und der Symptombelastung sind als die Struktur. In Folgeuntersuchungen sollten diese Zusammenhänge auf unterschiedlichen Strukturniveaus überprüft werden, da es möglich ist, dass die Ergebnisse durch Jugendliche mit zu geringem Strukturniveau verzerrt werden, d.h., dass die Struktur einen Mediationseffekt auf den Zusammenhang der Konfliktskalen mit den Outcome-Maßen hat. Dies ist in der OPD-KJ-2 insofern berücksichtigt, dass eine Konflikteinschätzung bei zu gering integriertem Strukturniveau nicht indiziert ist. Hier müssen Folgeuntersuchungen zeigen, für welche Strukturniveaus ein Selbstbericht reliabel und valide sein kann.

\section{Limitationen und Ausblick}

Wir berichten erste Ergebnisse mit der Selbst- und Fremdeinschätzung des Konfliktniveaus anhand eines Konfliktfragebogens, der für jugendliche Patient_innen und ihre Therapeut_innen entwickelt wurde. Die Ergebnisse sind ermutigend, ermöglichen sie doch eine Diskriminierung zwischen gesunden Jugendlichen und Patient_innen und zeigen eine ähnliche Einschätzung wie der Befundbogen an der gleichen Patientin bzw. am gleichen Patienten auf. Dies lässt darauf schließen, dass damit insgesamt eine ökonomische Erfassung des Konfliktniveaus möglich ist. Allerdings lassen die unbefriedigenden Beurteilerübereinstimmungen in einigen Konflikten darauf schließen, dass noch weiterer Forschungsbedarf notwendig ist. Dies gilt auch für die faktorenanalytischen Ergebnisse, die insgesamt unbefriedigend waren, wie auch schon für die Erwachsenenversion von Benecke et al. (2018) und die Version für Jugendliche von Hold et al. (2015) berichtet wurde. Man muss auch bedenken, dass die Erfassung durch 28 Items zwar ökonomisch ist, mit vier Items pro Konflikt jedoch die Varianz entsprechend gering ist. Hier wäre zu überlegen, ob nicht eine etwas längere Version bessere statistische Gütekriterien erbringen würde. Auch die diskriminante und Kriteriumsvalidität bedarf noch weiterer Forschungen. Insgesamt benötigen wir auch noch mehr Forschung an anderen klinischen Stichproben, z.B. stationären Patient_innen. Hier wird das Konfliktniveau möglicherweise anders ausfallen, auch werden möglicherweise andere Konflikte betroffen sein. Die Ergebnisse sind also stichprobenabhängig. Wir haben den Vergleich unterschiedlicher Einschätzmethoden, wegen des Fehlens von Videos im ambulanten Bereich, auf der Basis eines Videos einer stationären Patientin und unterschiedlicher Rater_innen durchgeführt, um eine einheitliche Informationsgrundlage für alle Rater_innen zu haben. Aufgrund der mehrfachen Einschätzung einer Patientin ist die Objektivität der zweiten Einschätzung eingeschränkt, was zu einer ähnlichen zweiten Einschätzung trotz anderer Form beitragen kann. Wünschenswert wären für die Zukunft hier Erhebungen und Auswertungen an Videos mit Patient_innen unterschiedlicher Störungen und aus ambulanten wie stationären Settings. Darüber hinaus kann es zu Reihenfolgeeffekten gekommen sein. Die Teilnehmer_innen der beiden Gruppen schätzen zwar in der unterschiedlichen Reihenfolge ein, dennoch ist dies nicht ganz auszuschließen und sollte in Folgeuntersuchungen bedacht werden. Des Weiteren sollte in künftigen Studien eine der Voraussetzungen für die Selbsteinschätzung bei Jugendlichen, das Strukturniveau, ebenfalls analysiert werden. $\mathrm{Zu}$ Beginn der Arbeit am Konfliktfragebogen und der Planung entsprechender Studien lag ein Strukturfragebogen für Kinder und Jugendliche noch nicht vor, er wurde erst 2019 publiziert (Schrobildgen et al., 2019). Wir verwendeten daher den Strukturfragebogen für Erwachsene. Es erscheint daher sinnvoll, für zukünftige Validierungen den Strukturfragebogen für Kinder und Jugendliche von Schrobildgen et al. (2019) einzusetzen und nach Alternativen für den RFQ zu suchen, der inzwischen in die Kritik geraten ist.

Die Arbeit zur Validierung des Konfliktfragebogens ist noch nicht abgeschlossen; der vorliegende Beitrag ist ein erster Versuch in die Richtung. Insgesamt zeigen die Befunde dieser ersten Validierung des Konfliktfragebogens, dass er in der Interraterübereinstimmung aus Therapeutensicht eine ebenso gute, teilweise sogar bessere Interraterübereinstimmung erbringt wie die bisherigen Ergebnisse zur Interraterreliabilität mit dem Befundbogen. Das sind ermutigende Ergebnisse in Bezug auf das Fremdrating. Auch was das Selbstrating angeht, sind die Ergebnisse bemerkenswert, zeigen sie doch die Möglichkeit einer Differenzierung zwischen gesunden Jugendlichen und $\mathrm{Pa}-$ tient_innen auf. Wenig zufriedenstellend sind, ähnlich wie in dem Konfliktfragebogen für Erwachsene (Benecke et al., 2018) und dem Konfliktfragebogen von Hold et al. (2015), die faktorenanalytischen Ergebnisse. Hier ist unbedingt weitere Forschung notwendig. Weitere Validierungsstudien sind erforderlich, die die Ergebnisse auf eine breitere Forschungsbasis stellen, dazu ist es hilfreich, wenn der Konfliktfragebogen im klinischen Alltag eingesetzt wird. 


\section{Elektronisches Supplement}

Das elektronische Supplement (ESM) ist mit der OnlineVersion dieses Artikels verfügbar unter

https://doi.org/10.1024/1422-4917/a000822.

ESM 1. Screeplots der Eigenwerte der explorativen Faktorenanalysen der Therapeuten-( $\mathrm{N}=46)$ und der Jugendlichenversionen ( $\mathrm{N}=121)$ der Langversion der Konfliktskalen. ESM 2. T-Verteilung der Rohwerte der 14 Subskalen der Konfliktskalen mit Verarbeitungsmodus aus Jugendlichenund aus Therapeutensicht.

\section{Literatur}

Arbeitskreis OPD-KJ-2. (Hrsg.). (2020). OPD-KJ-2: Operationalisierte Psychodynamische Diagnostik im Kindes- und Jugendalter. Grundlagen und Manual (3., unveränderte Aufl.). Bern: Hogrefe.

Benecke, C. \& Brauner, F. (2017). Motivation und Emotion. Psychologische und psychoanalytische Perspektiven. Stuttgart: Kohlhammer.

Benecke, C., Henkel, M., Doering, S., Jakobsen, T., Stasch, M., Dahlbender, R. et al. (2018). Der OPD-Konfliktfragebogen. Zeitschrift für Psychosomatische Medizin und Psychotherapie, 64, 380-393.

Bowlby, J. (1969). Bindung. Eine Analyse der Mutter-Kind-Beziehung. München: Kindler.

Cohen, J. (1988). Statistical power analysis for the behavioral sciences. Hillsdale: Erlbaum.

Ehrenthal, J.C., Dinger, U., Schauenburg, H., Horsch, L., Dahlbender, R.W. \& Gierk, B. (2015). Entwicklung einer 12-Item-Version des OPD-Strukturfragebogens (OPD-SFK). Zeitschrift für Psychosomatische Medizin und Psychotherapie, 61, 262-274.

Erikson, E.H. (1966). Identität und Lebenszyklus. Frankfurt a.M.: Suhrkamp.

Fonagy, P., Luyten, P., Moulton-Perkins, A., Lee, Y.W., Warren, F., Howard, S. et al. (2016). Development and validation of a selfreport measure of mentalizing: The Reflective Functioning Questionnaire. PLOS ONE, 11, e0158678

Freud, S. (1916/1917). Vorlesungen zur Einführung in die Psychoanalyse. In S. Freud, Gesammelte Werke, Band XI. Frankfurt a. M.: Fischer.

Hold, S., Benecke, C. \& Schüßler, G. (2015). Narzisstische Selbstdarstellung - Generation Me? Zeitschrift für Psychosomatische Medizin und Psychotherapie, 61, 36-42.

Kohut, H. (1979). Die Heilung des Selbst. Frankfurt a.M.: Suhrkamp.

Kornhas, L.A., Schröder-Pfeifer, P., Georg, A., Zettl, M. \& Taubner, S. (2020). Prozess des Mentalisierens in einer mentalisierungsbasierten Langzeittherapie für Borderline-Patienten: Eine Fallstudie. Psychotherapeut, 65, 357-365.

Rathgeber, M., Sommer, T. \& Seiffge-Krenke, I. (2014). Die Achse Konflikt der Operationalisierten Psychodynamischen Diagnostik im Kindes- und Jugendalter: Reliabilität und klinische Validität. Kinderanalyse, 22, 26-47.

Schrobildgen, C., Goth, K., Weissensteiner, R., Lazari, O. \& Schmeck, K. (2019). Der OPD-KJ2-SF - Ein Instrument zur Erfassung der Achse Struktur der OPD-KJ-2 bei Jugendlichen im Selbsturteil. Zeitschrift für Kinder- und Jugendpsychiatrie und Psychotherapie, 47, 428-440.
Seiffge-Krenke, I. (2020a). Jugendliche in der Psychodynamischen Psychotherapie: Kompetenzen für Diagnostik, Behandlungstechnik, Konzepte und Qualitätssicherung. Stuttgart: Klett-Cotta.

Seiffge-Krenke, I. (2020b). Die Jugendlichen und ihre Suche nach dem neuen Ich - Identitätsentwicklung in der Adoleszenz. Stuttgart: Kohlhammer.

Seiffge-Krenke, I., Dietrich, H., Adler-Corman, P., Timmermann, H., Rathgeber, M., Winter, S. et al. (2014). Die Konfliktachse der OPD-KJ-2: Ein Fallbuch für die klinische Arbeit. Göttingen: Vandenhoeck \& Ruprecht.

Seiffge-Krenke, I., Fliedl, R. \& Katzenschläger, P. (2013). Diagnosespezifische Strukturdefizite. Konsequenzen für die psychotherapeutische Behandlung von Kindern und Jugendlichen. Psychotherapeut, 58, 15-23.

Seiffge-Krenke, I., Mayer, S., Rathgeber, M. \& Sommer, T. (2013). Konflikt- und Strukturachse der Operationalisierten Psychodynamischen Diagnostik des Kindes- und Jugendalters. Hilfe bei Indikation und Therapieplanung. Psychotherapeut, 58, 6-14.

Seiffge-Krenke, I., Mayer, S. \& Winter, S. (2011). Beurteilerübereinstimmung bei der OPD-KJ: Wovon hängt sie ab und welchen Erfolg bringt das Training? Klinische Diagnostik und Evaluation, 4,176-193.

Stefini, A., Reich, G., Horn, H., Winkelmann, K., Ohmes, U., Frost, U. et al. (2013). Interrater-Reliabilität der OPD-KJ-Achsen Konflikt und Struktur. Praxis der Kinderpsychologie und Kinderpsychiatrie, 62, 255-269.

Winter, S., Jelen, A., Pressel, C., Lenz, K. \& Lehmkuhl, U. (2011). Klinische und empirische Befunde zur OPD-KJ. Praxis der Kinderpsychologie und Kinderpsychiatrie, 60, 41-59.

Woerner, W., Becker, A., Friedrich, C., Rothenberger, A., Klasen, H. \& Godman, R. (2002). Normierung und Evaluation der deutschen Elternversion des Strengths and Difficulties Questionnaire (SDQ): Ergebnisse einer repräsentativen Felderhebung. Zeitschrift für Kinder- und Jugendpsychiatrie und Psychotherapie, 30, 105-112.

Zimmermann, J., Stasch, M., Grande, T., Schauenburg, H. \& Cierpka, M. (2014). Der Beziehungsmuster-Q-Sort (OPD-BQS): Ein Selbsteinschätzungsinstrument zur Erfassung von dysfunktionalen Beziehungsmustern auf Grundlage der Operationalisierten Psychodynamischen Diagnostik. Zeitschrift für Psychiatrie, Psychologie und Psychotherapie, 62, 43-53.

\section{Historie}

Manuskript eingereicht: 19.10.2020

Nach Revision angenommen: 13.06.2021

Onlineveröffentlichung: 10.09.2021

\section{Interessenkonflikt}

Es bestehen keine Interessenkonflikte.

\section{Förderung}

Open-Access-Veröffentlichung ermöglicht durch die JohannesGutenberg-Universität Mainz.

\section{Prof. Dr. Inge Seiffge-Krenke}

Kostheimer Landstr. 11

55246 Mainz-Kostheim

Deutschland

seiffge-krenke@uni-mainz.de 
1. Frage: Wie viele Skalen/Konflikte werden in dem neu entwickelten Fragebogen zur Selbst- und Fremdeinschätzung auf Basis der OPD-KJ-2 unabhängig vom Verarbeitungsmodus eingeschätzt?

(Einfachauswahl)
a. 4
b. 5
c. 6
d. 7
e. 8

2. Frage: Was ist kein Konflikt nach OPD-KJ-2? (Einfachauswahl)

a. Schuldkonflikt

b. Identitätskonflikt

c. Selbstwertkonflikt

d. Ödipaler Konflikt

e. Interpersoneller Konflikt

3. Frage: Was beschreibt der Nähe-Distanz Konflikt nach der OPD-KJ-2 im aktiven Modus? (Einfachauswahl)

a. Angst vor Nähe und übersteigerte emotionale Unabhängigkeit

b. Angst vor Trennung und ständige Suche nach engen Beziehungen

c. Freude an offenen Räumen und Bewegung

d. Häufige Konflikte mit den eigenen Eltern

e. Häufige Impulsdurchbrüche
4. Frage: Wozu kann ein multiperspektivischer Fragebogen über die Konfliktachse für die Altersstufe 3 nach OPD-KJ-2 dienen? (Einfachauswahl)

a. Die Jugendlichen fühlen sich besser

b. Standardisierte Erfassung intrapsychischer Konflikte und ökonomischere Einschätzung

c. Insbesondere Behandlungen im Vorschulalter profitieren davon

d. Die intrapsychische Struktur wird erhoben

e. Die Beziehungsachse wird mit dem Patienten zusammen exploriert

\section{Frage: In der Einschätzung welcher beiden} intrapsychischen Konflikte unterscheiden sich jugendliche Patienten am deutlichsten von ihren gesunden Peers? (Einfachauswahl)

a. Schuldkonflikt und Konflikt Selbst versorgen vs. versorgt werden

b. Nähe-Distanz Konflikt und Schuldkonflikt

c. Nähe-Distanz Konflikt und ödipaler Konflikt

d. Identitätskonflikt und Selbstwertkonflikt

e. Konflikt Unterwerfung vs. Kontrolle und Identitätskonflikt 
Um Ihr CME-Zertifikat zu erhalten (min. drei richtige Antworten), schicken Sie bitte den ausgefüllten Fragebogen mit einem frankierten Rückumschlag bis zum 29.10.2021 an die nebenstehende Adresse. Später eintreffende Antworten und solche ohne bzw. mit nicht frankierten Rückumschlägen können nicht mehr berücksichtigt werden.

\section{Milena Becker}

LWL-Universitätsklinik Hamm der Ruhr-Universität Bochum Klinik für Kinder- und Jugend psychiatrie, Psychotherapie und Psychosomatik

Heithofer Allee 64

59071 Hamm, Deutschland

\section{Fortbildungszertifikat}

Die Ärztekammer Niedersachsen erkennt hiermit

2 Fortbildungspunkte an.

\section{Stempel}

Kinder- und Jugend-

psychiatrie und

Psychotherapie

05/2021

\section{(v) hogrefe}

Datum
„Konflikt-Fragebogen zur Selbst- und

Fremdeinschätzung“

Die Antworten bitte deutlich ankreuzen!
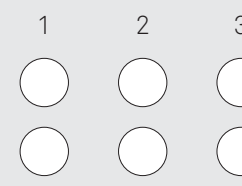

C

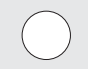

d

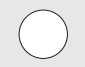

e

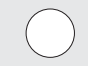

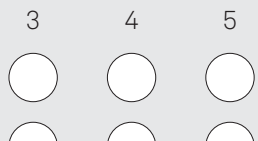
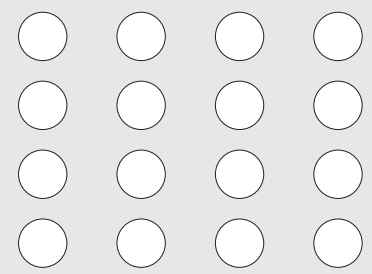

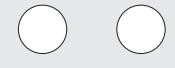

00

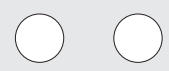

Ich versichere, alle Fragen ohne fremde Hilfe beantwortet zu haben.

Name

Berufsbezeichnung, Titel

Straße, Nr.

PLZ, Ort 